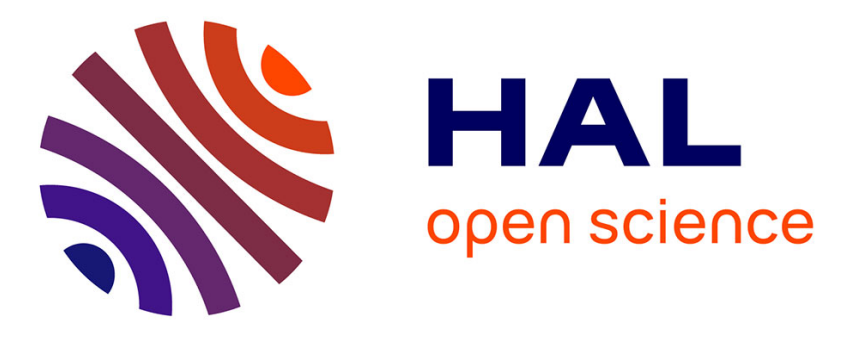

\title{
Detecting global bridges in networks
}

Pablo Jensen, Matteo Morini, Márton Karsai, Tommaso Venturini, Alessandro

Vespignani, Mathieu Jacomy, Jean-Philippe Cointet, Pierre Mercklé, Eric

Fleury

\section{- To cite this version:}

Pablo Jensen, Matteo Morini, Márton Karsai, Tommaso Venturini, Alessandro Vespignani, et al.. Detecting global bridges in networks. Journal of Complex Networks, 2015, 4 (3), pp.319-329. 10.1093/comnet/cnv022 . hal-01206166

\section{HAL Id: hal-01206166 https://inria.hal.science/hal-01206166}

Submitted on 1 Oct 2015

HAL is a multi-disciplinary open access archive for the deposit and dissemination of scientific research documents, whether they are published or not. The documents may come from teaching and research institutions in France or abroad, or from public or private research centers.

$$
\text { Copyright }
$$

L'archive ouverte pluridisciplinaire HAL, est destinée au dépôt et à la diffusion de documents scientifiques de niveau recherche, publiés ou non, émanant des établissements d'enseignement et de recherche français ou étrangers, des laboratoires publics ou privés. 
IMA Journal of Complex Networks (2015) Page 1 of 15 doi:10.1093/comnet/xxxxxx

\section{Detecting global bridges in networks}

PABLO JENSEN*

IXXI, Institut Rhonalpin des Systèmes Complexes, ENS Lyon; Laboratoire de Physique, UMR 5672, ENS Lyon 69364 Lyon, France

${ }^{*}$ Corresponding author: pablo.jensen@ens-lyon.fr

MATTEO MORINI

IXXI, Institut Rhonalpin des Systèmes Complexes, ENS Lyon; LIP, INRIA, UMR 5668, ENS de Lyon 69364 Lyon, France

MÁRTON KARSAI

IXXI, Institut Rhonalpin des Systèmes Complexes, ENS Lyon; LIP, INRIA, UMR 5668, ENS de Lyon 69364 Lyon, France

TOMmaso VENTURINI Médialab, Sciences Po, Paris

ALESSANDRO VESPIGNANI

MoBS, Northeastern University, Boston MA 02115 USA; ISI Foundation, Turin 10133, Italy

MathieU JACOMY

Médialab, Sciences Po, Paris

JEAN-PHILIPPE COINTET

Université Paris-Est, SenS-IFRIS

PIERRE MERCKLÉ

Centre Max Weber, UMR 5283, ENS Lyon 69364 Lyon, France

ERIC FLEURY

IXXI, Institut Rhonalpin des Systèmes Complexes, ENS Lyon; LIP, INRIA, UMR 5668, ENS de Lyon 69364 Lyon, France

[Received on XX XX XXXX; revised on XX XX XXXX; accepted on XX XX XXXX] 
The identification of nodes occupying important positions in a network structure is crucial for the understanding of the associated real-world system. Usually, betweenness centrality is used to evaluate a node capacity to connect different graph regions. However, we argue here that this measure is not adapted for that task, as it gives equal weight to "local" centers (i.e. nodes of high degree central to a single region) and to "global" bridges, which connect different communities. This distinction is important as the roles of such nodes are different in terms of the local and global organisation of the network structure. In this paper we propose a decomposition of betweenness centrality into two terms, one highlighting the local contributions and the other the global ones. We call the latter bridgeness centrality and show that it is capable to specifically spot out global bridges. In addition, we introduce an effective algorithmic implementation of this measure and demonstrate its capability to identify global bridges in air transportation and scientific collaboration networks.

Keywords: Centrality Measures, Betweenness Centrality, Bridgeness Centrality

JXX, JYY

\section{Introduction}

Although the history of graphs as scientific objects begins with Euler's [10] famous walk across Königsberg bridges, the notion of 'bridge' has rarely been tackled by network theorists' ${ }^{1}$. Among the few articles that took bridges seriously, the most famous is probably Mark Granovetter's paper on The Strength of Weak Ties [14]. Despite the huge influence of this paper, few works have remarked that its most original insights concern precisely the notion of 'bridge' in social networks. Granovetter suggested that there might be a fundamental functional difference between strong and weak ties. While strong ties promote homogeneous and isolated communities, weak ties foster heterogeneity and crossbreeding. Or, to use the old tönnesian cliché, strong ties generate Gemeinshaft, while weak ties generates Gesellshaft [8]. Although Granovetter does realize that bridging is the phenomenon he is looking after, two major difficulties prevented him from a direct operationalization of such concept: "We have had neither the theory nor the measurement and sampling techniques to move sociometry from the usual small-group level to that of larger structures" (ibidem, p. 1360). Let's start from "the measurement and sampling techniques". In order to compute the bridging force of a given node or link, one needs to be able to draw a sufficiently comprehensive graph of the system under investigation. Networks constructed with traditional ego-centered and sampling techniques are too biased to compute bridging forces. Exhaustive graphs of small social groups will not work either, since such groups are, by definition, dominated by bounding relations. Since the essence of bridges is to connect individuals across distant social regions, they can only be computed in large and complete social graphs. Hopeless until a few years ago, such endeavor seems more and more reasonable as digital media spread through society. Thanks to digital traceability it is now possible to draw large and even huge social networks [20, 30, 31].

Let's discuss now the second point, the "theory" needed to measure the bridging force of different edges or nodes ${ }^{2}$. Being able to identify bounding and bridging nodes has a clear interest for any type of network. In social networks, bounding and bridging measures (or "closure" and "brokerage", to use Burt's terms [6]) tell us which nodes build social territories and which allow items (ideas, pieces of information, opinions, money...) to travel through them. In scientometrics' networks, these notions tell us which authors define disciplines and paradigms and which breed interdisciplinarity. In ecological

\footnotetext{
${ }^{1}$ We refer to the common use of the word 'bridge', and not to the technical meaning in graph theory as 'an edge whose deletion increases its number of connected components'

${ }^{2}$ In this paper, we will focus on defining the bridgeness of nodes, but our definition can straightforwardly be extended to edges, just as the betweenness of edges is derived from that of nodes.
} 
networks, they identify relations, which create specific ecological communities and the ones connecting them to larger habitats.

In all these contexts, it is the very same question that we wish to ask: do nodes or edges reinforce the density of a cluster of nodes (bounding) or do they connect two separated clusters (bridging)? Formulated in this way, the bridging/bounding question seems easy to answer. After having identified the clusters of a network, one should simply observe if a node connects nodes of the same cluster (bounding) or of different clusters (bridging). However, the intra-cluster/inter-cluster approach is both too dependent on the method used to detect communities and flawed by its inherent circular logic: it uses clustering to define bridging and bounding ties when it is precisely the balance of bridges and bounds that determines clusters. Remark that, far from being a mathematical subtlety, this question is a key problem in social theory. Defining internal (gemeinschaft) and external (gesellschaft) relations by presupposing the existence and the composition of social groups is absurd as groups are themselves defined by social relations.

In this paper, we introduce a measure of bridgeness of nodes that is independent on the community structure and thus escapes this vicious circle, contrary to other proposals [7, 24]. Moreover, since the computation of bridgeness is straightforwardly related to that of the usual betweenness, Brandes' algorithm [5] can be used to compute it efficiently ${ }^{3}$. To demonstrate the power of our method and identify nodes acting as local or global bridges, we apply it on a synthetic network and two real ones: the world airport network and a scientometric network.

\section{Measuring bridgeness}

Identifying important nodes in a network structure is crucial for the understanding of the associated real-world system [3, 4, 9], for a review see [25]. The most common measure of centrality of a node for network connections on a global scale is betweenness centrality $(B C)$, which "measures the extent to which a vertex lies on paths between other vertices" $[11,12]$. We show in the following that, when trying to identify specifically global bridges, $B C$ has some limitations as it assigns the same importance to paths between the immediate neighbours of a node as to paths between further nodes in the network. In other words $\mathrm{BC}$ is built to capture the overall centrality of a node, and is not specific enough to distinguish between two types of centralities: local (center of a community) and global (bridge between communities). Instead, our measure of bridging is more specific, as it gives a higher score to global bridges. The fact that $B C$ may attribute a higher score to local centers than to global bridges is easy to see in a simple network (Figure 1). The logics is that a "star" node with degree $k$, i.e. a node without links between all its first neighbors (clustering coefficient 0 ) receives automatically a $B C=$ $k(k-1) / 2$ arising from paths of length 2 connecting the node's first neighbors and crossing the central node. More generally, if there exist nodes with high degree but connected only locally (to nodes of the same community), their betweenness may be of the order of that measured for more globally connected nodes. Consistent with this observation, it is well-known that for many networks, $B C$ is highly correlated with degree $[13,23,26]$. A recent scientometrics study tried to use betweenness centrality as "an indicator of the interdisciplinarity of journals" but noted that this idea only worked "in local citation environments and after normalization because otherwise the influence of degree centrality dominated the betweenness centrality measure [21].

To avoid this problem and specifically spot out global centers, we decompose $B C$ into a local and a

\footnotetext{
${ }^{3}$ We have written a plug-in for Gephi [1] that computes this measure on large graphs. See Supplementary Informations for a pseudo-algorithm for both node and edge bridgeness.
} 


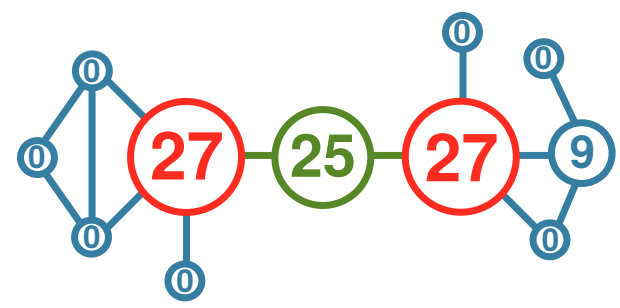

(a) Betweenness centrality

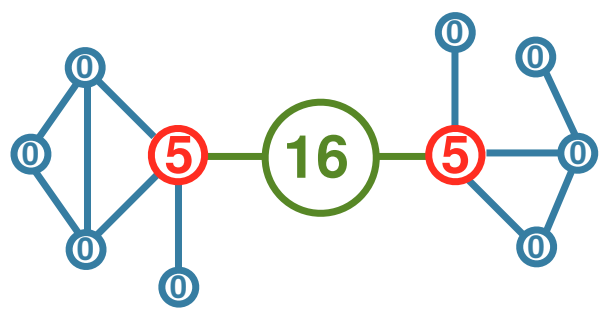

(b) Bridgeness centrality

FIG. 1. The figures show the betweenness (a) and bridgeness (b) scores for a simple graph. Betweenness does not distinguish centers from bridges, as it attributes a slightly higher score (Figure a, scores $=27$ ) to high-degree nodes, which are local centers, than to the global bridge (Figure a, score $=25$ ). In contrast, bridgeness rightly spots out the node $($ Figure $b$, score $=16)$ that plays the role of a global bridge.

global term, the latter being called 'bridgeness' centrality. Since we want to distinguish global bridges from local ones, the simplest approach is to discard shortest paths, which either start or end at a node's first neighbors from the summation to compute $B C$ (Eq. 1.1). This completely removes the paths that connect two non connected neighbors for 'star nodes' (see Figure 1) and greatly diminishes the effect of high degrees, while keeping those paths that connect more distant regions of the network.

More formally in a graph $\mathscr{G}=(V, E)$, where $V$ assigns the set of nodes and $E$ the set of links the definition of the betweenness centrality for a node $j \in V$ stands as:

$$
B C(j)=\operatorname{Bri}(j)+\operatorname{local}(j),
$$

where

$$
\begin{aligned}
B C(j) & =\sum_{i \neq j \neq k} \frac{\sigma_{i k}(j)}{\sigma_{i k}} \\
\operatorname{Bri}(j) & =\sum_{i \notin N_{G}(j) \wedge k \notin N_{G}(j)} \frac{\sigma_{i k}(j)}{\sigma_{i k}} \\
\operatorname{local}(j) & =\sum_{i \in N_{G}(j) \vee k \in N_{G}(j)} \frac{\sigma_{i k}(j)}{\sigma_{i k}} .
\end{aligned}
$$

Here the summation runs over any distinct node pairs $i$ and $k$; $\sigma_{i k}$ represents the number of shortest paths between $i$ and $k$; while $\sigma_{i k}(j)$ is the number of such shortest paths running through $j$. Decomposing $B C$ into two parts (right hand side) the first term defines actually the global term, bridgeness centrality, where we consider shortest paths between nodes not in the neighbourhood of $j\left(N_{G}(j)\right)$, while the second local term considers the shortest paths starting or ending in the neighbourhood of $j$. This definition also demonstrates that the bridgeness centrality value of a node $j$ is always smaller or equal to the corresponding $B C$ value and they only differ by the local contribution of the first neighbours. Fig. 1 illustrates the ability of bridgeness to specifically highlight nodes that connect different regions of a graph. Here the $B C$ (Fig. 1a) and bridgeness centrality values (Fig. 1b) calculated for nodes of the same network demonstrate that bridgeness centrality gives the highest score to the node which is central globally (green), while $B C$ does not distinguish among local or global centers, and actually assigns the highest score to nodes with high degrees (red). 
In the following, to further explore the differences between these measures we define an independent reference measure of bridgeness using a known partitioning of the network. This measure provides us an independent ranking of the bridging power of nodes, that we correlate with the corresponding rankings using the $B C$ and bridgeness values. In addition we demonstrate via three example networks that bridgeness centrality is always more specific than $B C$ to identify global bridges.

\section{Computing global bridges from a community structure}

To identify the global bridges independently from their score in $B C$ or bridgeness, we use a simple indicator inspired by the well-known Rao-Stirling index [17, 27-29], as this indicator is known to quantify the ability of nodes to connect different communities. Moreover, it includes the notion of "distance", which is important for distinguishing local and global connections. However, we note that this index needs as input a prior categorization of the nodes into distinct communities. Our global indicator $G$ in Eq.1.3 for node $i$ is defined as:

$$
G(i)=\sum_{J \in \text { communities }} l_{I J} \delta_{i, J}
$$

where the sum runs over communities $J$ (different from the community of node $i$, taken as $I$ ), $\delta_{i, J}$ being 1 if there is a link between node $i$ and community $J$ and 0 otherwise. Finally, $l_{I J}$ corresponds to the 'distance' between communities $I$ and $J$, as measured by the inverse of the number of links between them: the more links connect two communities, the closer they are. Nodes that are only linked to nodes of their own community have $G=0$, while nodes that connect two (or more) communities have a strictly positive indicator. Those nodes that bridge distant communities, for example those that are the only link between two communities, have high $G$ values.

As a next step we use this reference measure (i.e. the global indicator) to rank nodes and compare it to the rankings obtained by the two tentative characteristics of bridging ( $B C$ and bridgeness) in three large networks.

\section{Synthetic network: unbiased LFR}

We start with a synthetic network obtained by a method similar to that of Lancichinetti et al [18]. This method leads to the so-called 'LFR' networks with a clear community structure, which allows to easily identify bridges between communities. We have only modified the algorithm to obtain bridges without the degree bias which arises from the original method. Indeed, LFR first creates unconnected communities and then chooses randomly internal links that are reconnected outside the community. This leads to bridges, i.e. nodes connected to multiple communities, which have a degree distribution biased towards high degrees. In our method, we avoid this bias by randomly choosing nodes, and then one of their internal links, which we reconnect outside its community as in LFR. As reference, we use the global indicator defined above. As explained, this indicator depends on the community structure, which is not too problematic here since, by construction, communities are clearly defined in this synthetic network.

Fig. 3a shows that bridgeness provides a ranking that is closer to that of the global indicator than $B C$. Indeed, we observe that the ratio for bridgeness is higher than for $B C$. This means that ordering nodes by their decreasing bridgeness leads to a better ranking of the 'global' scores - as measured by $\mathrm{G}$ - than the corresponding ordering by their decreasing $B C$ values. As shown in the simpler example of a 1000-node network (demonstrated in Fig. 2), $B C$ fails because it ranks too high some nodes that have 


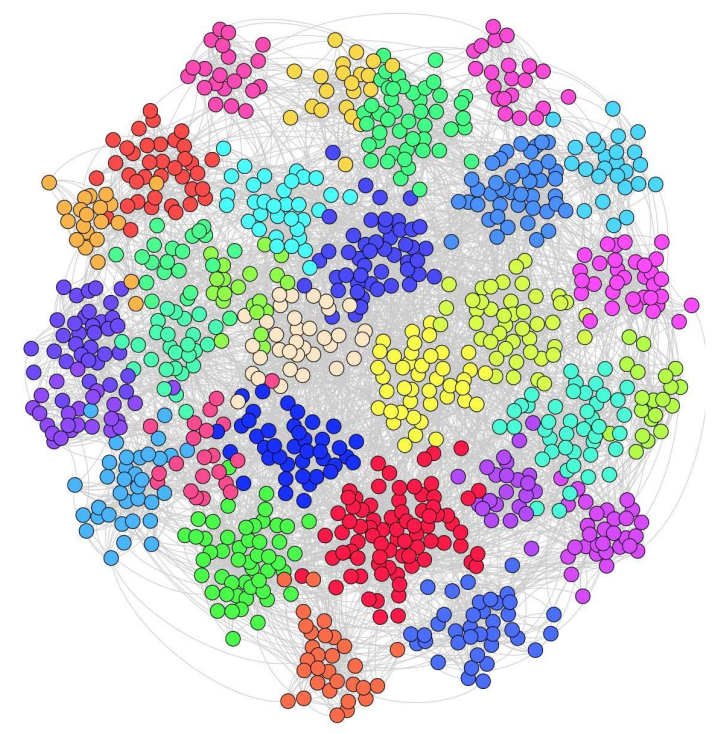

FIG. 2. Artificial network with a clear community structure using Lancichinetti et al [18] method. For clarity, we show here a smaller network containing 1000 nodes, 30 communities, 7539 links (20\% inter-and 80\% intra-community links). Each color corresponds to a community as detected by modularity optimization $[2,25]$.

no external connection but have a high degree. A detailed analysis of the nodes of a cluster is given in Supplementaty Informations.

In addition we directly measured $\langle\text { locterm }\rangle_{i}(k)=\langle(B C(i, k)-B r i(i, k)) / B C(i, k)\rangle_{i}$, the average relative contribution of the local term in $B C$ for nodes of the same degree (see Fig. 3b). We observe a negative correlation, which means that the local term is dominating for low degree nodes, while high degree nodes have higher bridgeness value as they have a higher chance to connect to different communities.

\section{Real network 1: airport's network}

Proving the adequacy of bridgeness to spot out global bridges on real networks is more difficult, because generally communities are not unambiguously defined, therefore neither are global bridges. Then, it is difficult to show conclusively that bridgeness is able to specifically spot these nodes. To answer this challenge, our strategy is the following:

(i) We use flight itinerary data providing origin destination pairs between commercial airports in the world (International Air Transport Association). The network collects 47,161 transportation connections between 7,733 airports. Each airport is assigned to its country.

(ii) We consider each country to be a distinct 'community' and compute a global indicator based on this partitioning, as it allows for an objective (and arguably relevant) partition, independent from any community detection methods. Then we show that bridgeness offers a better ranking than BC to identify airports that act as global bridges, i.e. that connect countries internationally. 

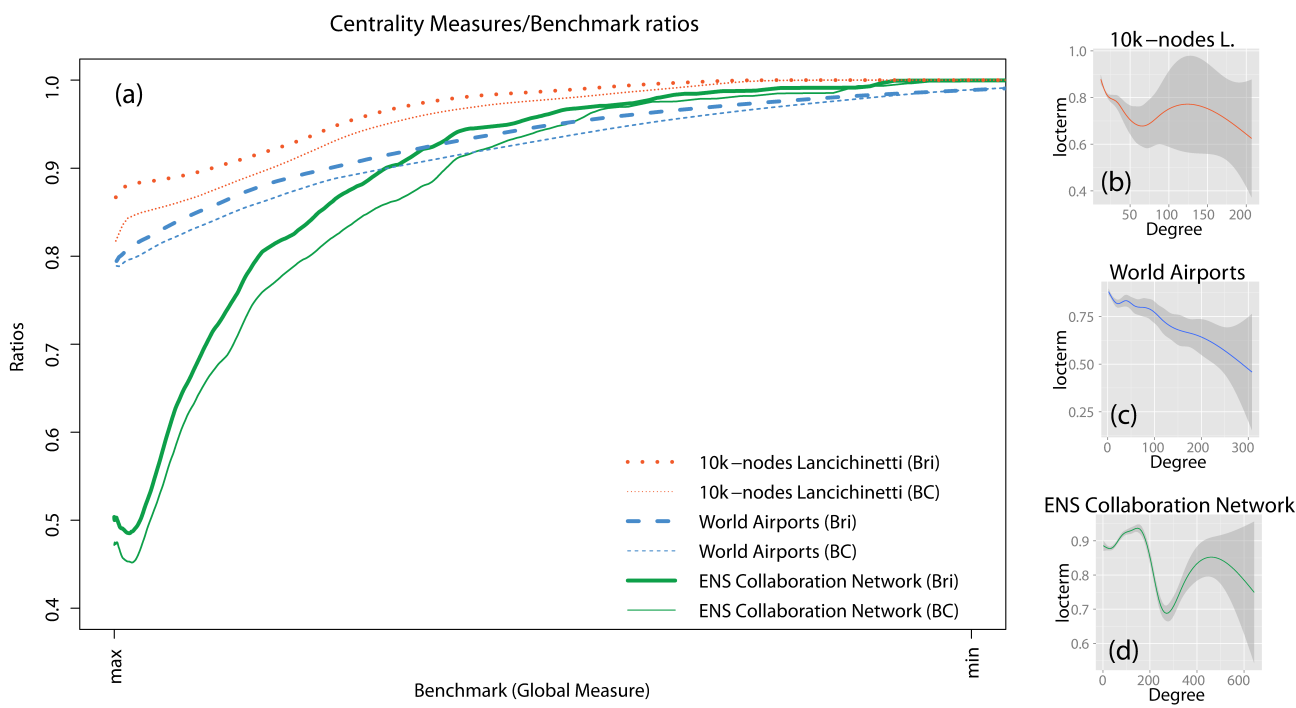

FIG. 3. (a) Ability of $\mathrm{BC}$ or bridgeness to reproduce the ranking of bridging nodes, taking as reference the global indicator (Eq 2). For each of the three networks, we first compute the cumulative sums for the global measure G, according to three sorting options: the $\mathrm{G}$ measure itself and the two centrality metrics, namely $\mathrm{BC}$ and bridgeness. By construction, sorting by $\mathrm{G}$ leads to the highest possible sum, since we rank the nodes starting by the highest $\mathrm{G}$ score and ending by the lowest. Then we test the ability of $\mathrm{BC}$ or bridgeness to reproduce the ranking of bridging nodes by computing the respective ratios of their cumulative sum, ranking by the respective metric (BC or Bri), to the cumulative obtained by the $\mathrm{G}$ ranking. A perfect match would therefore lead to a ratio equal to 1 . Since we observe that the ratio for bridgeness is higher than for $\mathrm{BC}$, this means that ordering nodes by their decreasing bridgeness leads to a better ranking of the 'global' scores as measured by G. To smooth the curves, we have averaged over 200 points. Curves corresponding to different networks are colorised as LFR (red), Airports (blue), ENS (green). (b, c, d): average relative local terms as function of node degree for the three investigated networks (for definition see text). 


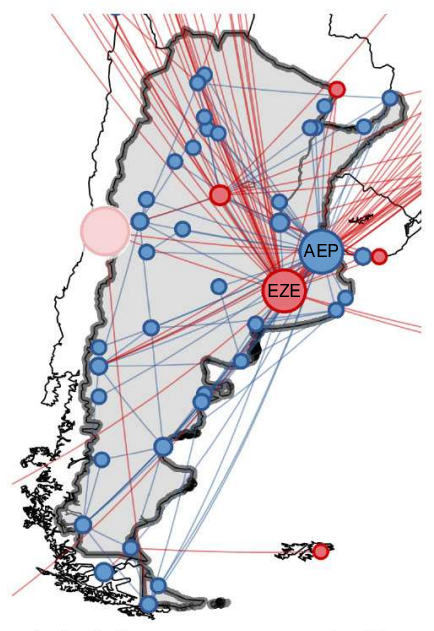

(a) Betweenness centrality

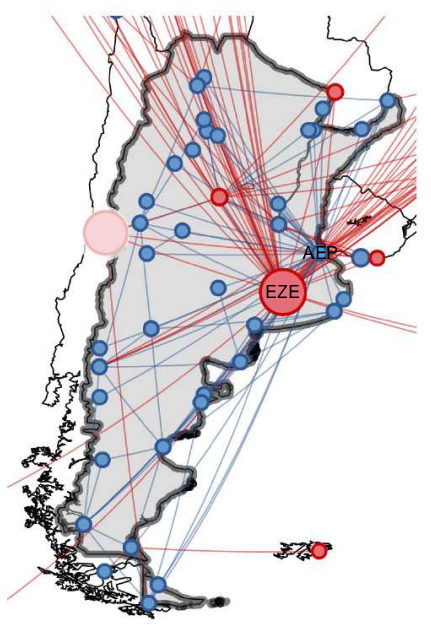

(b) Bridgeness centrality

FIG. 4. Example of the two largest Argentinean airports, Ezeiza (EZE) and Aeroparque (AEP). Both have a similar degree (54 and 45 respectively), but while the first connects Argentina to the rest of the world (85\% of international connections, average distance 2.848 miles, $\mathrm{G}=2327.2$ ), Aeroparque is only a local center ( $18 \%$ of international connections, average distance 570 miles, $\mathrm{G}=9.0$ ). However, as in the simple graph (Figure 1), BC gives the same score to both $\left(B C_{E Z E}=79,000\right.$ and $\left.B C_{A E P}=82,000\right)$, while bridgeness clearly distinguishes the local center and the bridge to the rest of the world, by attributing to the global bridge a score 250 times higher $\left(B r i_{E Z E}=46,000\right.$ and $\left.B r i_{A E P}=174\right)$. Red nodes represent international airports while blue nodes are domestic.

As an example, in Fig. 4 we show the two largest airports of Argentina, Ezeiza (EZE) and Aeroparque (AEP). Both have a similar degree (54 and 45 respectively), but while the first connects Argentina to the rest of the world, Aeroparque mostly handles domestic flights, thus functioning as a local center. This is confirmed by the respective G values: 2327.2 (EZE) and 9.0 (AEP). However, just like in our simple example in Fig. 1, $B C$ gives the same score to both, while bridgeness clearly distinguishes between the local domestic center and the global international bridge by attributing to the global bridge a score 250 times higher (see Fig. 4). This can partly be explained by the fact that AEP is a 'star' node (low clustering coefficient: 0.072 ), connected to 12 very small airports, for which it is the only link to the whole network. All the paths starting from those small airports are cancelled in the computation of the bridgeness (they belong to the 'local' term in Eq.1.1), while $B C$ counts them equally as any other path.

More generally, Figure 3 shows that, as for the Airport network, bridgeness provides again a ranking that is closer to that of the global indicator. Indeed, ordering nodes by their decreasing bridgeness leads to a ranking that is closer to the ranking obtained by the global score than the ranking by decreasing $B C$. In addition we found again negative correlations between the average relative local term and node degrees (see Fig. 3c), assigning similar roles for low and high degree nodes as in case of the synthetic network.

\section{Real network 2: scientometric network of ENS Lyon}

The second example of a real network is a scientometric graph of a scientific institution [15], the "Ecole normale supérieure de Lyon" (ENS, see Figure 5). This networks adds authors to the usual co-citation 
network, as we want to understand which authors connect different sub-fields and act as global, interdisciplinary bridges. To identify the different communities, we rely on modularity optimization [2], which leads to a relevant community partition because scientific networks are highly structured by disciplinary boundaries. This is confirmed by the high value of modularity generated by this partition (0.89). In Figure 5, the authors of different communities are shown with different colors, and their size corresponds to their betweenness (left) or bridgeness (right) centrality, which clearly leads to highlight different authors as the main global bridges, which connect different subfields. We compute the Stirling indicator (Eq.1.1) based on the modularity structure to identify the global bridges. As for the previous networks, Fig. 3 shows that bridgeness ranks the nodes in a closer way than $B C$ to the ranking provided by the global measure based on community partition. On the other hand the corresponding $\langle$ locterm $\rangle(k)$ function (see Fig. 3d) suggests a slightly different picture in this case. Here nodes with large but moderate degrees (smaller than $\sim 200$ ) have high local terms suggesting that they act as local centres, while nodes with higher degrees have somewhat smaller local terms assigning their role to act as global bridges.

\section{Discussion}

In this paper we introduced a measure to identify nodes acting as global bridges in complex network structures. Our proposed methodology is based on the decomposition of $B C$ into a local and global term, where the local term considers shortest paths that start or end at one of the node's neighbors, while the global term, what we call bridgeness, is more specific to identify nodes which are globally central. We have shown, on both synthetic and real networks, that the proposed bridgeness measure improves the capacity to specifically find out global bridges as it is able to distinguish them from local centers. One crucial advantage of our measure of bridgeness over former propositions is that it is independent of the definition of communities.

However, the advantage in using bridgeness depends the precise topology of the network, and mainly on the degree distribution of bridges as compared to that of all the nodes in the network. When bridges are high-degree nodes, $B C$ and bridgeness give an equally good approximation, since high-degree bias do not play an important role in this case. Instead, when some bridges have low degrees, while some high-degree nodes act like local centers of their own community, bridgeness is more effective to identify bridges as $B C$ gives equally high rank to nodes with high degree, even if they are not connected to nodes outside of their community. We demonstrated that bridgeness is systematically more specific to spot out global bridges in all the networks we have studied here. Although the improvement was small on average, typically 5 to $10 \%$, even a small amelioration of a widely used measure is in itself an interesting result.

We should also note that, except on simple graphs, comparing these two measures is difficult since there is no clear way to identify, independently, the 'real' global bridges. We have used community structure when communities seem clear-cut, but then we fall into the circularity problems stressed in the introduction. Using metadata on the nodes (i.e. countries for the airports) may solve this problem but raises others, as metadata do not necessarily correspond to structures obtained from the topology of the network, as shown recently on a variety of networks [16]. Another possible extension would be to identify overlapping communities to identify independently global bridges, as nodes involved in multiple communities, and correlate them with the actual measure, which provides a direction for future studies. However, in any case identifying global bridges remains a difficult problem as it is tightly linked to another difficult problem, that of community detection. Decomposing $B C$ into a local and a global term helps to improve the solution, but many questions remain still open for further inquiry. 

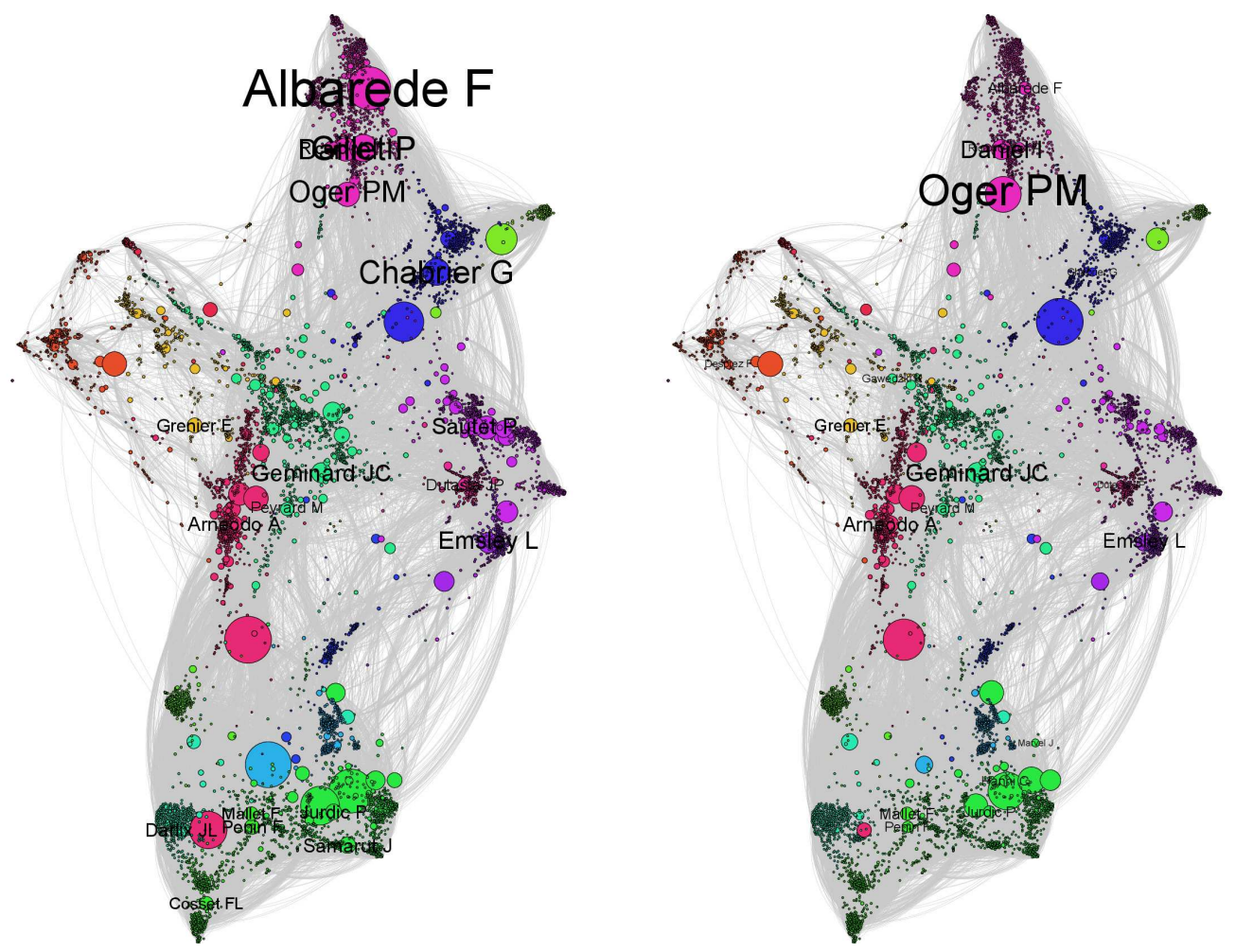

FIG. 5. Co-citation and co-author network of articles published by scientists at ENS de Lyon. Nodes represent the authors or references appearing in the articles, while links represent co-appearances of these features in the same article. The color of the nodes corresponds to the modularity partition and their size is proportional to their $B C$ (left) or to their bridgeness (right), which clearly leads to different rankings (references cited are used in the computations of the centrality measures but appear as dots to simplify the picture). We only keep nodes that appear on at least four articles and links that correspond to at least 2 co-appearances in the same paper. After applying these thresholds, the 8000 articles lead to 8883 nodes (author or references cited in the 8000 articles) and 347,644 links. The average degree is 78 , the density 0.009 and the average clustering coefficient is 0.633 . Special care was paid to avoid artifacts due to homonyms. Weights are attributed to the links depending on the frequency of co-appearances (cosine distance, see [15]. 


\section{REFERENCES}

1. Bastian, M., Heymann, S. \& Jacomy, Q. (2009) Gephi: An Open Source Software for Exploring and Manipulating Networks. International AAAI Conference on Weblogs and Social Media.

2. Blondel, V. D., Guillaume, J.-L., Lambiotte, R. \& Lefebvre, E. (2008) Fast unfolding of communities in large networks. J. Stat. Mech, 10, P10008.

3. Bonacich, P. (1987) Power and centrality: A family of measures. American journal of sociology, 92(5), 11701182.

4. Borgatti, S. P. (2005) Centrality and network flow. Social networks, 27(1), 55-71.

5. Brandes, U. (2001) A faster algorithm for betweenness centrality. Journal of Mathematical Sociology, 25(2), 163-177.

6. Burt, R. S. (2005) Brokerage and closure: An introduction to social capital. Oxford University Press, Oxford.

7. Cheng, X.-Q., Ren, F.-X., Shen, H.-W. \& Zhang, Z.-K. (2010) Bridgeness: a local index on edge significance in maintaining global connectivity. Journal of Statistical Mechanics: Theory and Experiment, 2010(10), P10011.

8. Coser, R. (1975) The Complexity of Roles as Seedbed of Individual Autonomy.. In Coser, L., editor, The Idea of Social Structure: Essays in Honor of Robert Merton. Harcourt Brace Jovanovich, New York.

9. Estrada, E. \& Rodriguez-Velazquez, J. A. (2005) Subgraph centrality in complex networks. Physical Review E, 71(5), 056103.

10. Euler, L. (1741 (1736)) Solutio problematis ad geometriam situs pertinentis. Commentarii academiae scientiarum Petropolitanae, 8, 128-140.

11. Freeman, L. C. (1977) A set of measures of centrality based on betweenness. Sociometry, pages 35-41.

12. Freeman, L. C. (1979) Centrality in social networks conceptual clarification. Social networks, 1(3), 215-239.

13. Goh, K.-I., Oh, E., Kahng, B. \& Kim, D. (2003) Betweenness centrality correlation in social networks. Physical Review E, 67(1), 017101.

14. Granovetter, M. S. (1973) The strength of weak ties. American journal of sociology, 78(6), 1360-1380.

15. Grauwin, S. \& Jensen, P. (2011) Mapping scientific institutions. Scientometrics, 89(3), 943-954.

16. Hric, D., Darst, R. K. \& Fortunato, S. (2014) Community detection in networks: Structural communities versus ground truth. Physical Review E, 90(6), 062805.

17. Jensen, P. \& Lutkouskaya, K. (2014) The many dimensions of laboratories interdisciplinarity. Scientometrics, 98(1), 619-631.

18. Lancichinetti, A., Fortunato, S. \& Radicchi, F. (2008) Benchmark graphs for testing community detection algorithms. Physical review E, 78(4), 046110.

19. Latour, B., Jensen, P., Venturini, T., Grauwin, S. \& Boullier, D. (2012) The whole is always smaller than its parts-a digital test of Gabriel Tardes' monads. The British journal of sociology, 63(4), 590-615.

20. Lazer, D., Pentland, A. S., Adamic, L., Aral, S., Barabási, A.-L., Brewer, D., Christakis, N., Contractor, N., Fowler, J., Gutmann, M., Jebara, T., King, G., Macy, M., Roy, D. \& Van Alstyne, M. (2009) Life in the network: the coming age of computational social science. Science, 323(5915), 721-723.

21. Leydesdorff, L. (2007) Betweenness centrality as an indicator of the interdisciplinarity of scientific journals. Journal of the American Society for Information Science and Technology, 58(9), 1303-1319.

22. Louf, R., Jensen, P. \& Barthelemy, M. (2013) Emergence of hierarchy in cost-driven growth of spatial networks. Proceedings of the National Academy of Sciences, 110(22), 8824-8829.

23. Nakao, K. (1990) Distribution of measures of centrality: enumerated distributions of Freeman's graph centrality measures. Connections, 13(3), 10-22.

24. Nepusz, T., Petróczi, A., Négyessy, L. \& Bazsó, F. (2008) Fuzzy communities and the concept of bridgeness in complex networks. Physical Review E, 77(1), 016107.

25. Newman, M. (2010) Networks: an introduction. Oxford University Press.

26. Newman, M. E. J. (2005) A measure of betweenness centrality based on random walks. Social networks, 27(1), 39-54.

27. Rafols, I. (2014) Knowledge Integration and Diffusion: Measures and Mapping of Diversity and Coherence. 
In Ding, Y., Rousseau, R. \& Wolfram, D., editors, Measuring Scholarly Impact, pages 169-190. Springer.

28. Rao, C. R. (1982) Diversity and dissimilarity coefficients: a unified approach. Theoretical Population Biology, 21(1), 24-43.

29. Stirling, A. (2007) A general framework for analysing diversity in science, technology and society. Journal of the Royal Society Interface, 4(15), 707-719.

30. Venturini, T. \& Latour, B. (2010) The social fabric: Digital traces and quali-quantitative methods. Proceedings of Future En Seine 2009, pages 87-101.

31. Vespignani, A. (2009) Predicting the behavior of techno-social systems. Science, 325(5939), 425. 


\section{Supplementary Informations}

\section{S1. Modified Brandes algorithm}

Bridgeness algorithm, inspired by Brandes" "faster algorithm" [5]

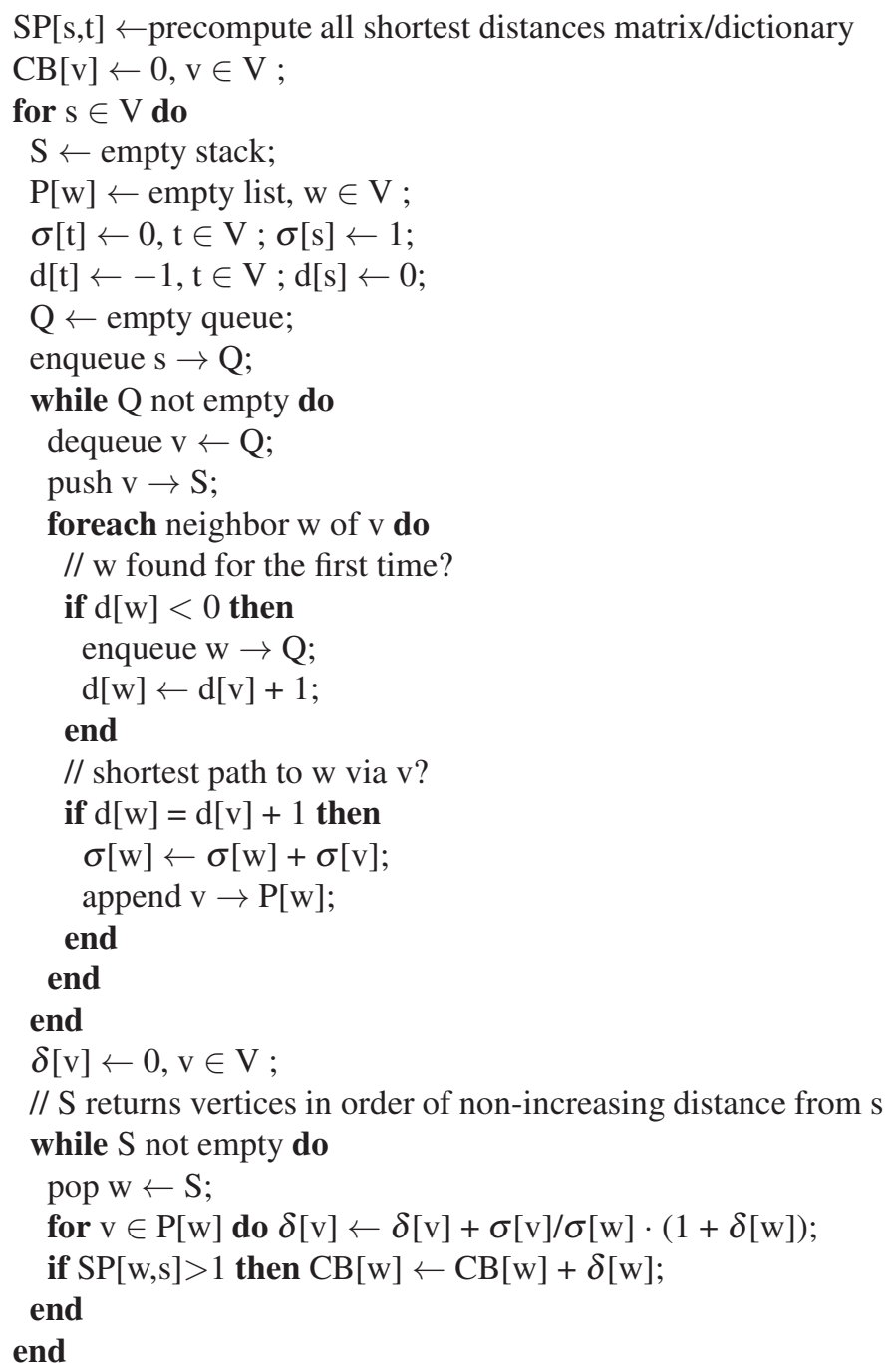

\section{S2. Case study on a synthetic network community}

The specificity of bridgeness and the influence of the degree, which prevents BC from identifying correctly the most important bridges, can be exemplified by examining the scores of nodes in cluster 5 of the synthetic network. This cluster is linked to cluster 13 by 5 connections (through nodes 248,861 , 471,576 and 758 ) and to cluster 1 by a single connection (through node 232). BC gives roughly the 


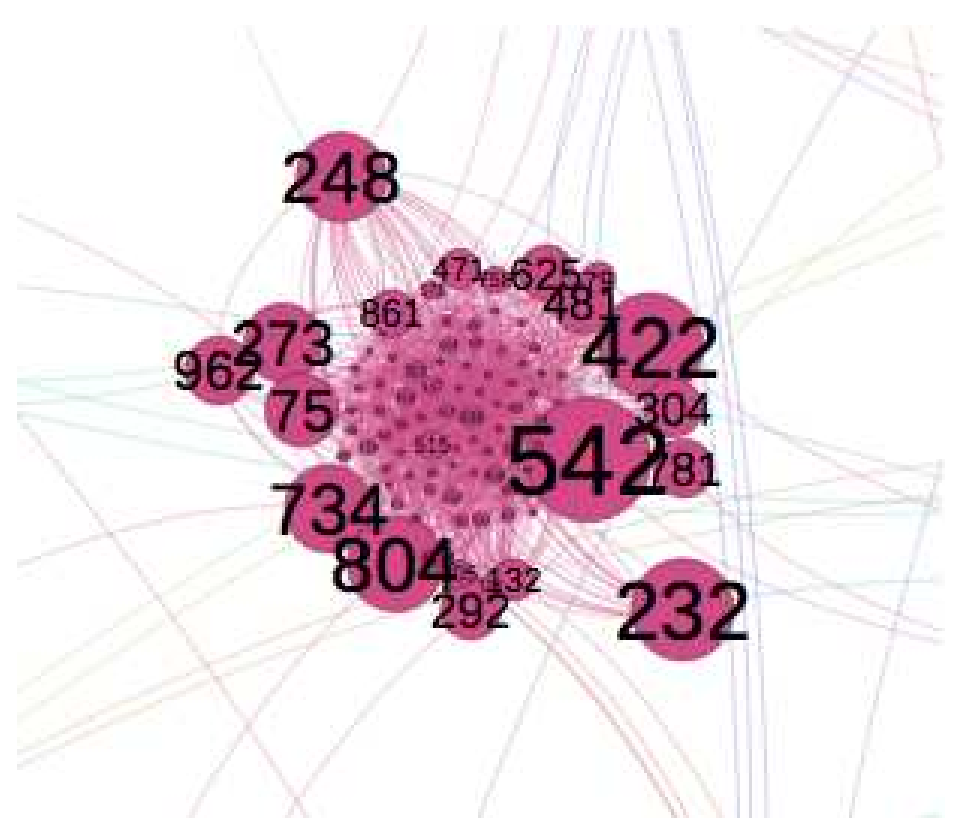

FIG. S1. Zoom on cluster 5 of the synthetic network. The numbers show node's labels, while the size of the nodes is proportional to their $\mathrm{BC}$ score.

same score to nodes 232 and 248, while bridgeness attributes a score almost 4 times higher to node 232, correctly pointing out the importance of this single bridge between clusters 5 and 1 . This is because BC is confused by the high degree of node 248 (41) as compared to node 232 low degree (20). Therefore, by counting all the shortest paths, BC attributes too high a bridging score to node 248 . Second problem with $\mathrm{BC}$, it gives a high score to nodes that are not connected to other communities, merely because they are local centers, i.e. they have a high degree. For example, node 515 obtains a higher BC score than node 758 (Table S1), even if node 515 has no connection to other communities (but degree 49), contrary to node 758 (connected to cluster 5, but degree 23). Bridgeness never ranks higher local centers than global bridges: here, it correctly assigns a 5 times higher score to node 758 than to node 515 . 
Table S1. Nodes in community 5 of the synthetic network, ranked by decreasing BC (see text)

\begin{tabular}{l||r|r|r|r|r} 
Id & Stirling & Modularity Class & Betweenness & Bridgeness & Degree \\
\hline 542 & 0.0222 & 5 & 9173.71 & 2644.62 & 44 \\
422 & 0.0278 & 5 & 7714.27 & 3855.62 & 35 \\
232 & 0.0950 & 5 & 7551.22 & 5846.86 & 20 \\
804 & 0.0285 & 5 & 6995.63 & 2824.64 & 34 \\
248 & 0.0082 & 5 & 6588.65 & 1624.30 & 48 \\
734 & 0.0907 & 5 & 6410.31 & 4373.72 & 21 \\
273 & 0.0322 & 5 & 5698.28 & 2631.59 & 30 \\
75 & 0.0868 & 5 & 5349.47 & 3558.31 & 22 \\
962 & 0.0399 & 5 & 4989.66 & 2951.45 & 24 \\
292 & 0.0399 & 5 & 4377.77 & 1939.06 & 24 \\
481 & 0.0256 & 5 & 4305.68 & 1796.92 & 25 \\
781 & 0.0475 & 5 & 4257.93 & 2200.21 & 20 \\
304 & 0.0434 & 5 & 4221.64 & 2467.65 & 22 \\
625 & 0.0202 & 5 & 3964.21 & 1314.62 & 32 \\
861 & 0.0108 & 5 & 3295.01 & 714.44 & 36 \\
132 & 0.0200 & 5 & 2985.45 & 1157.49 & 24 \\
471 & 0.0154 & 5 & 2865.07 & 1296.38 & 25 \\
79 & 0.0302 & 5 & 2256.02 & 1004.28 & 21 \\
205 & 0.0208 & 5 & 1921.65 & 788.51 & 23 \\
515 & 0.0000 & 5 & 1884.07 & 86.45 & 49 \\
758 & 0.0166 & 5 & 1791.80 & 435.66 & 23 \\
608 & 0.0200 & 5 & 1777.54 & 522.75 & 24
\end{tabular}

